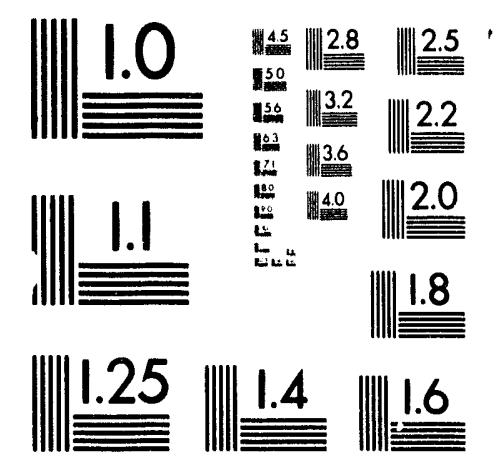



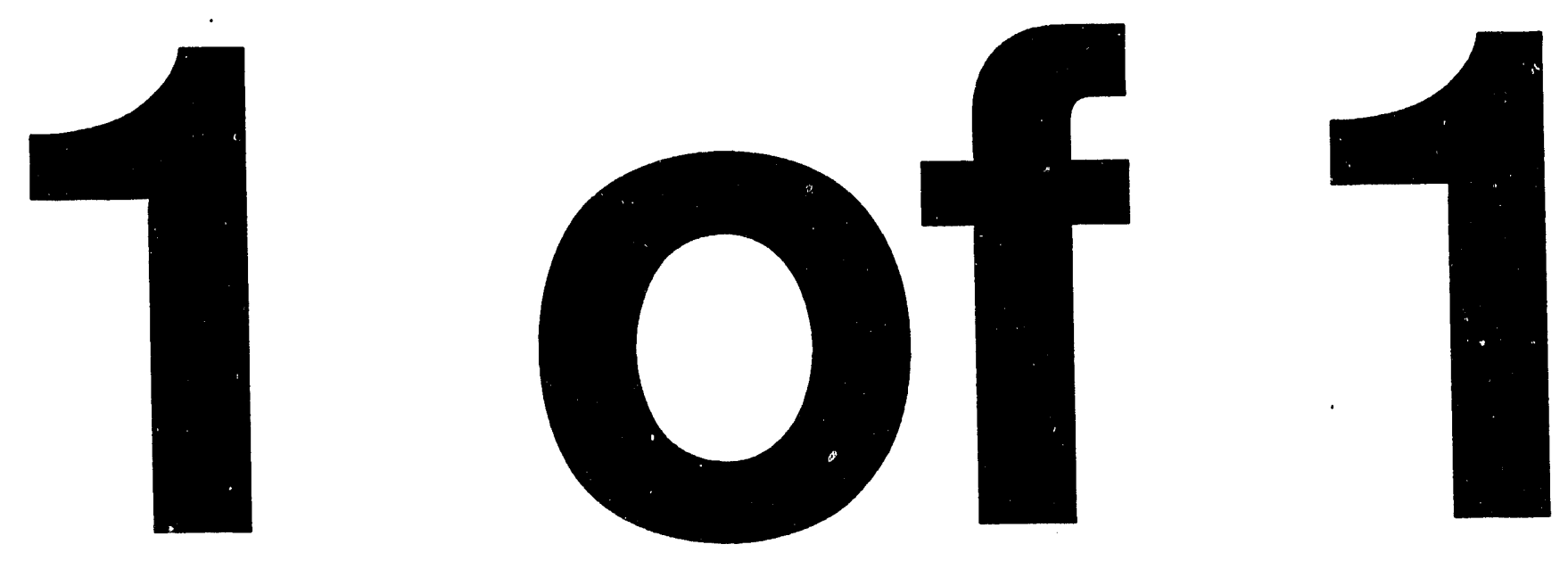


\title{
Gamma Large Area Silicon Telescope (GLAST): \\ Applying Silicon Strip Detector technology to the \\ Detection of Gamma Rays in Space*
}

\author{
W. B. Atwood \\ The GLAST Collaboration ${ }^{+}$ \\ Stanford Linear Accelerator Center \\ Stanford, CA 94309
}

\begin{abstract}
The recent discoveries and excitement generated by space satillite experiment EGRET (presently operating on Compton Gamma Ray Observatory - CGRO) have prompted an investigation into modern detector technologies for the next generation space based gamma ! ay telescopes. The GLAST proposal is based on silicon strip detectors as the "technology of choice" for space application: no consumables, no gas volume, robust (versus fragile), long lived, and self triggerable. The GLAST detector basically has two components: a tracking module preceding a calorimeter. The tracking module has planes of crossed strip $(x, y) 300$ um pitch silicon detectors coupled to a thin radiator to measure the coordinates of converted electron-positron pairs. The gap between the layers $(-5 \mathrm{~cm})$ provides a lever arm for track fitting resulting in an angular resolution of $<0.1^{\circ}$ at high energy. The status of this $R$ \& $D$ effort is discussed including details on triggering the instrument, the organization of the detector electronics and readout, and work on computer simulations to model this instrument.
\end{abstract}

Presented at GLAST Silicon Detector Conference

Hiroshima, Japan, May 22-24, 1993

\footnotetext{
.*Work supported by Department of Encrgy contract DE-AC03-76SF00515.

.'The GLAST Collaboration: Ying-Chi Lin, P.F. Michelson, P.L. Nolan (Physics Dept., Stanford University); W.B. Atwood, E. D. Bloom, G.L. Godfrey, A.E. Snyder, R.E. Taylor (Stanford Linear Accelerator Center, Stanford University); and P. L. Hertz, K. S. Wood (Naval Research Laboratory).
} 


\section{Introduction}

Extra-terrestrial gamma rays were first directly observed in 1962 with the launch of EXP X1 [1]. Only 31 events were detected, but sufficient to provide direct evidence that indeed high energy photons were present in the emissions from distant objects. The next data arrived in 1968 when OSO-3 was put into orbit. The data yield increased by an order of magnitude and established that most of the $\gamma s$ were coming from the plane of our galaxy. SAS- 2 was launched in 1972 and was the first to employ a triggered pair conversion telescope. It detected about 8000 ys over its 7 month lifetime and established that some of these high energy photons were coming from discreet sources. The COS-B experiment, launched three years later, employed similar technology adding a calorimeter capable of resolving energies up to $\sim 3 \mathrm{GeV}$. This experiment accumulated events over the next 7 years, increasing by over an order of magnitude the data previously available.

Late in the 1960s Bob Hofstadler and colleagues conceived a much larger gamma ray telescope [2]. The new instrument, named Egret, was initially scheduled to fly as part of HEO I, but its weight caused it to be reassigned to a spacecraft dedicated to observing $x$-rays and high energy $y s$. The spacecraft, the Compton Gamma Ray Observatory (or CGRO), was to be part of an early NASA Space Shuttle mission, but with delays and then the tragic Challenger launch in 1986, CGRO didn't get off the ground until 1991. Since then data has been successfully accumulated by all four experiments on board.

Egret has had a very successful initial data gathering period. Everything from unexpected solar flare phenomena, to hither to undiscovered pulsars, to new extra galactic high energy y sources have been discovered. It is beyond the scope of this presentation to do more then just scratch the surface of these new observations. These early Egret results are the motivation for continuing this progression of astrophysics experiments.

Figure 1 shows a schematic drawing of the Egret. It is obvious that the technologies employed were old even at the time of the initial proposal. Egret is comprised of three basic building blocks: 1) a pair conversion telescope made from triggered spark chambers interspersed with thin radiators; 2) a Nal calorimeter about 7.8 radiation lengths in depth; 3) and a triggering system consisting of an anti-coincident scintillator dome (veto) and a time-of-flight (TOF) system to establish directionality. The spark chamber is fired when there is "nothing" in the veto, the TOF has an acceptable timing signal, and there is at least $-35 \mathrm{MeV}$ of energy seen in the Nal. Magnetic cores are used to read out the resulting sparks. The data is then down linked to the ground where track finding is done on the pattern of read out hits. Finally, gamma rays are reconstructed from the pattern of observed tracks. Data acquisition will continue as long as the gas supply for the spark chambers lasts.

Since its launch in 1991, Egret has performed an "all sky survey" which is shown in Figure 2 [3]. The data used in the plot are for events with energies above $100 \mathrm{MeV}$, and the shading indicates the relative intensity, white being the highest. The bright band across this plot shows emissions from our galaxy, while the distinct points above and below the galactic plane are extra galactic sources (presumably these cover the sky quasi-uniformly but aren't seen in the plane of the galaxy, being overwhelmed by the more local signals). 
An interesting region of the sky is located at the far right in Fig. 2. Here, the two sources, Geminga (upper) and the Crab (lower), are both seen to be strong emitters of $>100 \mathrm{MeV}$ ys. During an early observing period in 1991, a surprise occurred and is shown in Fig. 3. Our sun produced a strong solar flare [4] within the field of view (not usually a problem as the Sun isn't normally an intense source of high energy photons). Figure 3 shows this dramatic event as the Sun suddenly became an intense source of $\sim 2 \mathrm{GeV}$ ys.

Until Egret, Geminga wasn't known to be a high energy y emitter, and it had only been weakly seen in the $x$-ray region. The Egret data now shows it to be an intense source of high energy $\gamma \mathrm{s}$, in fact brighter then the Crab at high energy. Local intensity maps of the sky in this region cut on different $\gamma$ energy bands shown in Fig. 4 illustrate this feature [5]. For energies above $1 \mathrm{GeV}$, Geminga appears to be much brighter then the Crab. Also, it is well known that the Crab has a pulsar at its core. Figure 5 shows that Geminga [6] is also a pulsed source, an Egret discovery. What is truly remarkable about this pulsar is that it isn't seen in either the optical or radio regions of the spectrum.

Beyond our galaxy, Egret has found about two dozen sources [7]. Most of these sources were here-to unknown to have emissions in the high energy end of the spectrum. Many correlate with known objects, but some do not. The data acquisition from Egret is now nearing the end of its second year. The mission is projected to last about another four years.

\section{The GLAST R\&D Project}

The successes of Egret, some of which have been discussed above, provide strong motivation for a second generation "Egret." Improvements in the following areas are clearly desirable: 1) larger acceptance and higher efficiency, 2) improved angular resolution of reconstructed $\gamma s$, 3) extended energy range, and 4) a long lifetime. The GLAST (Gamma ray Large Area Silicon Telescope) R \& D program's goal is to produce a proposal for the next instrument in this series. GLAST was started a year ago in response to a NASA request for R \& D proposals. The Stanford side of the Egret collaboration had strong ties to close-by SLAC where interest in Astro-Physics already existed. Particle detection technology has progressed a long way from triggered spark chambers, and the silicon strip detecror technology might be a good solution to the Egret problem. A proposal was submitted and subsequently funded by NASA for one year in which a more detailed design study could be accomplished.

We adopted the design principle that GLAST technology would be an adaptation of existing HEP detectors. Silicon strip detectors would provide tracking with a modern crystal calorimeter (read out using silicon diodes) to provide the energy measurement. The maximum size of presently available silicon detectors is only $6 \mathrm{~cm} \times 6 \mathrm{~cm}$, but by ganging together three (possibly four) such "tiles," the channel count may be kept to a reasonable level. A large device is easier to build if it can be made modular, and we opted to arrange GLAST as a mosaic of semi-autonomous tower modules. The rest of the design concept followed logically from Egret and is shown in Figure 6.

Early in the discussions, it was determined that the large area silicon detectors covering the aperture could "double" as an $\mathrm{x}$-ray detector. A coded aperture (forming a multi pin-hole camera) placed in front of these veto layers could provide an $x$-ray imager of unparalleled area and acceptance. No more will be said about this aspect of the 
GLAST project as we have yet to investigate the consequence for $y$ detection of placing this material well within the field of view.

The GLAST design is made from 100 tower modules (see Fig. 6), giving an entrance aperture of $3.28 \mathrm{~m}^{2}$. Egret's aperture is $0.66 \mathrm{~m}^{2}$. Also, GLAST doesn't have a time-of-flight (TOF) system for triggering. The pattern recognition in the silicon tracker will provide excellent veto capability for backgrounds. The absence of the TOF gives GLAST a much better aspect ratio then might otherwise be achieved, and this directly translates into a larger solid angle coverage.

GLAST doesn't have separate veto detectors covering its sides. Again, using the pattern of hits in the silicon seems to be sufficient to determine whether or not an event "materialized" (pair conversion in the case of $\gamma$ ) within the tracker. The simulations indicate that we can accept events entering the sides provided that they track through at least two layers of silicon before converting. This further extends the solid angle coverage albeit with diminishing projected area: GLAST has excellent peripheral vision. A comparison between the angular coverage of GLAST and Egret is shown in Fig. 7. Here the settings Egret took to produce the previously shown "all-sky-survey" (Fig. 2) are shown by the many solid circles. The large open circle centered on $\left(0^{\circ}, 0^{\circ}\right)$ gives the equivalent GLAST angular coverage, while the lightly shaded gray annulus around it shows the extent of the above mentioned peripheral vision. Furthermore, over the GLAST angular acceptance, the area of the instrument times efficiency is more then 10 times larger.

We have recently completed a simulation tool for studying GLAST built on the new C++ Monte Carlo program Gismo [8]. Electromagnetic interactions were "borrowed" from EGS4 [9], while hadronic interactions employ the Gheisha code[10]. A side view of GLAST with a $1 \mathrm{GeV}$ photon converting is shown in Fig. 8a. The blow-up of the conversion point in Fig. $8 \mathrm{~b}$ illustrates the level of detail in present simulation. To date we have verified the GLAST acceptance, the energy containment of the calorimeter, the rejection ability of the instrument for $\gamma$ s entering from the back as well as for high energy protons striking the calorimeter, and the angular resolution for reconstructed photon directions.

\section{Si Strip Detectors in Space}

Having covered the elements of the GLAST conceptual design above, we now discuss why we concluded that silicon strip technology is the "technology of choice" for space applications. Our list of perceived advantages includes: 1) NO consumables, 2) NO external trigger, 3) high efficiency, 4) excellent two hit resolution, 5) well defined coordinates, 6 ) long life time, and 7) fail-safe from many common mode failures.

The fact that GLAST won't need a gas supply as Egret does, is a major advantage. It contributes to a long life time and reduces common mode failures. Also, there is no pressure containment vessel. Items 3)-5) listed above are important in that the quality of the data that the pattern recognition software has to deal with is high. Very soon after the initial interaction, the separate tracks of the resulting $\mathrm{e}^{+}-\mathrm{e}^{-}$pair are resolvable. The initial conversion point is well 
defined, as the converters are almost in direct contact with the silicon detectors and the converter-detector layer is very thin. No trajectory-dependent corrections to this and the other coordinates on the track will be required.

A major departure from previous gamma ray space missions is the absence of components for triggering and vetoing background events. There is no encapsulating veto system since there isn't lots of material around to serve as a target for cosmic ray protons. Also, there is no TOF system. These systems are not required for triggering, as the silicon detectors are live all the time and can provide their own trigger. To accomplish this, it is envisioned to have a discriminator associated with each strip in conjunction with the usual amplifier. The "or'd" discriminator signal from an entire plane is used to alert a local micro-computer and subsequently read out the hit strips and their pulse heights. These events are to be time stamped allowing match ups with data from other planes in other towers. The event rates at the tower level are quite modest compared with current HEP requirements, and we don't forsee major difficulties. The import question with respect to the elimination of the TOF and anti-coincident shield is whether or not the hit information in the silicon tracker is sufficient to achieve good background rejection.

The principal technological challenges associated with the silicon strip technology are: 1) high channel count could require lots of power, 2) establishing this technology as space-qualified, and 3) cost. The answer to 1) is that present technology, driven by SSC needs, per channel pre-amp power of around $100 \mu \mathrm{W}$ is achievable [11]. Our present design has less then $2 \times 10^{6}$ channels, and thus would require a few hundred watts. This is well within the acceptable range for space craft.

To be space-qualified, this technology must not have hidden surprises when launched on a rocket and put into orbit. For example, much of "space-qualified" electronics must be double wire bonded in case a contact breaks during the high stress period during launch. This technology is well ahead of others in this respect as it is similar to the bulk of the electronics used in all space craft systems.

Finally, the cost issue. The price of large silicon strip detectors has come down by about one order of magnitude in the last ten years. A decade ago it would have been foolish to propose a device requiring almost $80 \mathrm{~m}^{2}$ of silicon strip detectors. Furthermore, when one considers the entire cost of a mission such as GLAST, the projected \$20-30 million for the silicon detectors [12] is a significant fraction but less than half of the launch cost.

\section{Conclusions}

The present Egret experiment has provided an exciting glimpse of the sky at high energy, and strongly suggests a larger, better instrumented device to carry on where it leaves off. The exciting results from Egret, some of which were shown at the beginning of the talk, when extrapolated to a GLAST scale instrument, could profoundly influence our knowledge of distance objects as wel'. as our overall picture of the universe. We have concluded that at least for the present, silicon strip detectors are the "technology of choice" for particle detection and tracking in the space environment. Furthermore, this existing technology, already well advanced in HEP, needs only be adapted to this new challenge. We are now in the early stages of simulating a design around the GLAST concept. While far from complete with respect to optimization issues, background rejection, etc., we are encouraged that this concept is indeed viable. 
Our program at Stanford over the next few years will be to construct a GLAST Tower module and then test it in the SLAC beam to validate the Monte Carlo simulation. At the same time, we will be beginning the process of building a collaboration, hopefully international in scope, to support, build, and utilize GLAST.

\section{References}

[1] Rodney Hillier, "Gamma Ray Astronomy," Clarendon Press, Oxford, ISBN 0-19-851451-4 (1984).

[2] During the first decade of this project much work was done in prototyping components and not until 1978 was the final proposal submitted to NASA.

[3] Science News 143260.

[4 ]G. Kanbach et al., Astronomy \& Astrophysics Supplement 97, (1993) 349.

[5] D. J. Thompson in "The Vancouver Meeting -- Particles and Fields ' 91 ," World Publishing, Singapore, (1992) 913, and P. L. Nolan et al., IEEE Trans. Nucl. Sci. 39, (1992) 993.

[6] D. L. Bertsch et al., Nature 357, ( 1992) 306.

[7] P. Michelson, private communication

[8] W. B. Atwood and T. H. Burnett, "Gismo: C++ Classes for HEP," Oriceedings of the Intr. Conf. on Cumputing in High Energy Physics '92, CERN 92-07, Dec. 1992. Also see W. Atwood et al, "The Gismo Project," C++ Reports, March-April 1993, Volume 5, No. 3, 38.

[9] W. R. Nelson, H. Hirayama, and W. O. Rogers, "The EGS4 Code Jystem," SLAC-Report-265, (1985).

[10] H. Fesefeldt, "Gheisha, the Simulation of Hadronic Showers - Physies and Applications," (2.6.12) PITHA 85/02 Also see K. Lassila, "Hadronic Cascade Simulations in GEANT," CERN, CN/9 1/03, (1991).

[11]Present designs for the SSC exceed $100 \mu \mathrm{W} /$ channel due to the req. speed. The GLAST application can use all CMOS technology which reduces power by a large factor: $100 \mu \mathrm{W} /$ channel seems well within reach. D. Dorfan (UCSC), private communication.

[12] Cost estimate based on priliminary discussions with Hamamatsu Corp.

( $\$ 780$ per $6 \times 6 \mathrm{sq} . \mathrm{cm}$ single sided detector) 


\section{Figure Captions}

Figure 1. Schematic drawing of Egret. Gamma rays convert in the trigger spark chamber stack and deposit energy in the Nal calorimeter. The TOF and anti-coincident dome aid in triggering and background rejection

Figure 2. The "all sky survey" performed by Egret for $\gamma s>100 \mathrm{MeV}$. The figure is shown in galactic co-ordinates: $\left(0^{\circ}, 0^{\circ}\right)$ is the galactic center while $\left(0^{\circ}, \pm 180^{\circ}\right)$ is the anti-center.

Figure 3. The patch of sky containing Geminga and the Crab: a) before a solar flare and b) during a solar flare.

Figure 4. Geminga and the Crab shown in three energy bands: a) 50-100 MeV (the Crab dominates), b) 100-300 MeV Geminga and the Crab appear at almost equal intensities, and c) $>1000 \mathrm{MeV}$ Geminga dominates.

Figure 5. The "light curve" for Geminga showing the pulsed nature of this source. The plot maps the full $360^{\circ}$ of phase onto the interval $(0,1)$. The full period is $247 \mathrm{msec}$.

Figure 6. The GLAST strawman design. In the silicon tracker, $\gamma$ s are converted, and their energies are measured by a Csl calorimeter. Note the absence of TOF and an encapsulating anti-coincidences dome.

Figure 7. Comparison of Egret's acceptance to GLAST's. The settings made by Egret to preform the "all sky survey" are shown by the small circles. One setting of GLAST is shown by the large circle centered on $\left(0^{\circ}, 0^{\circ}\right)$. The shaded area surrounding the central GLAST acceptance shows the extent of the edge acceptance (peripheral vision).

Figure 8. A $1 \mathrm{GeV} g$ event from the Gismo simulation of GLAST: a) side view of the entire detector and b) a close-up of the conversion point. The pulse beights in the silicon strips are shown by the bars drawn perpendicular to the detector planes, while the energy deposits in the calorimeter crystals are shown by the heights of the boxes.

\section{DISCLAIMER}

This report was prepared as an account of work sponsored by an agency of the United States Government. Neither the United States Gnvernment nor any agency thereof, nor any of their employees, makes any warranty, express or implied, or assumes any legal liability or responsibility for the accuracy, completeness, or usefulness of any information, apparatus, product, or process disclosed, or represents that its use would not infringe privately owned rights. Reference herein to any specific commercial product, process, or service by trade name, trademark, manufacturer, or otherwise does not necessarily constitute or imply its endorsement, recommendation, or favoring by the United States Government or any agency thereof. The views and opinions of authors expressed herein do not necessarily state or reflect those of the United States Government or any agency thereof. 


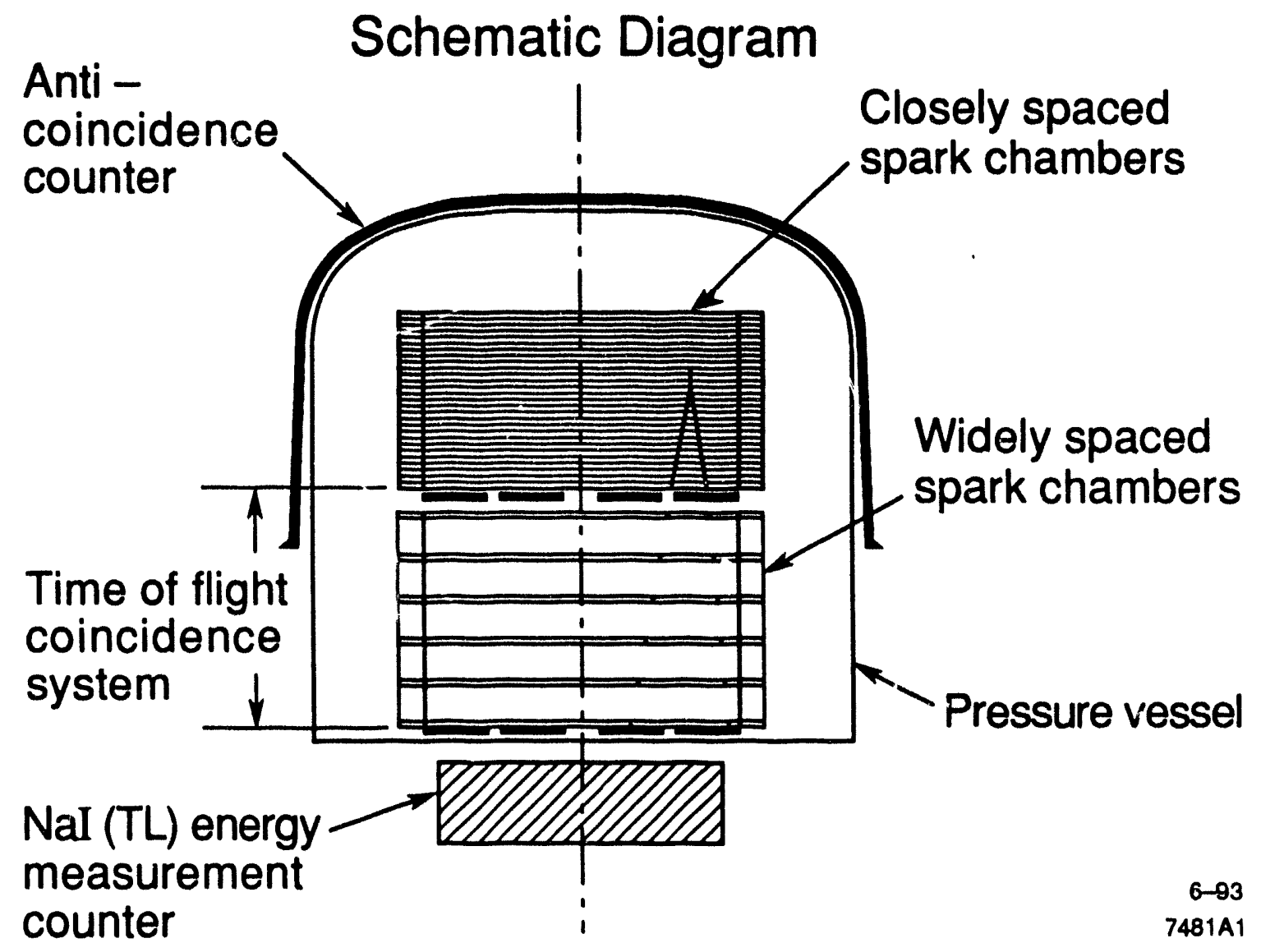

Fig. 1 


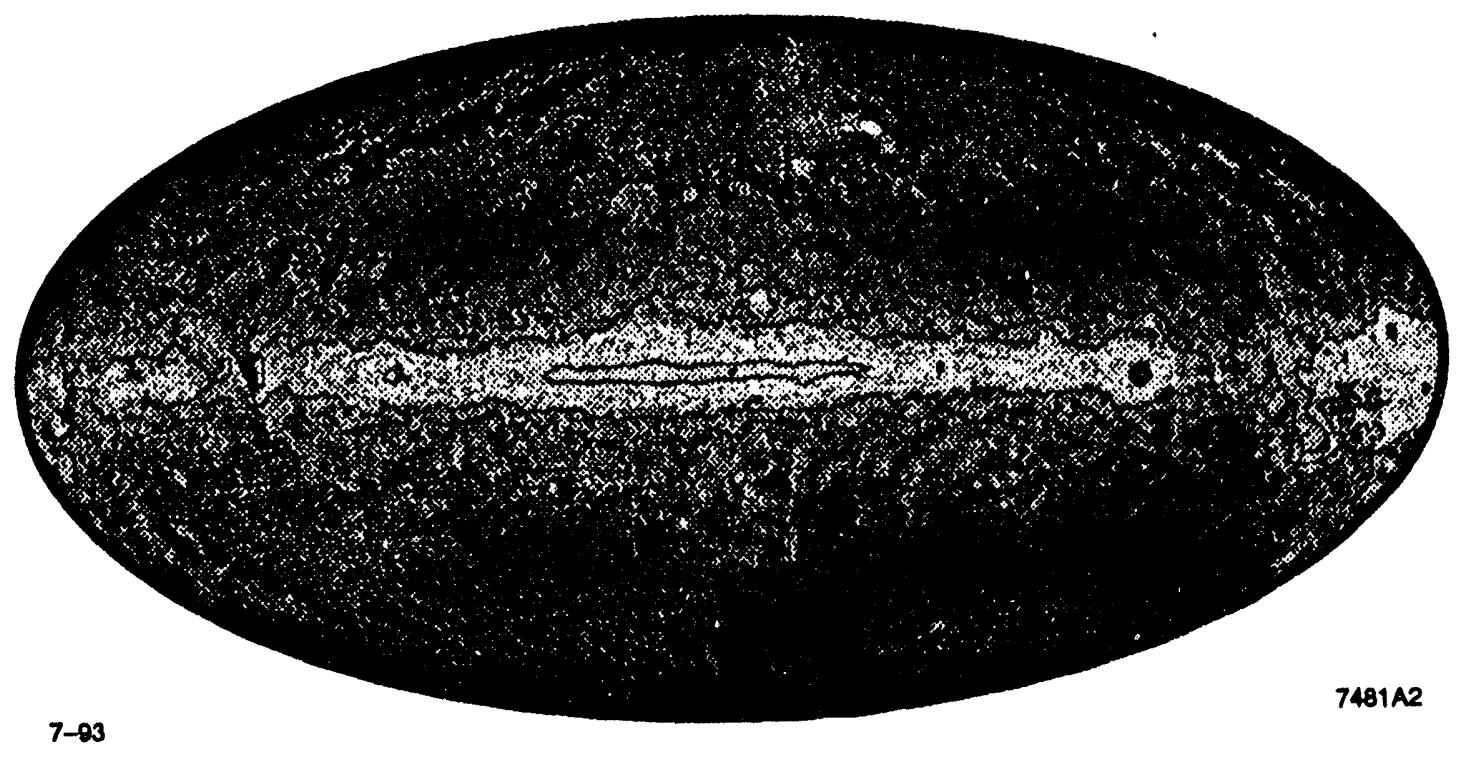

Fig. 2 


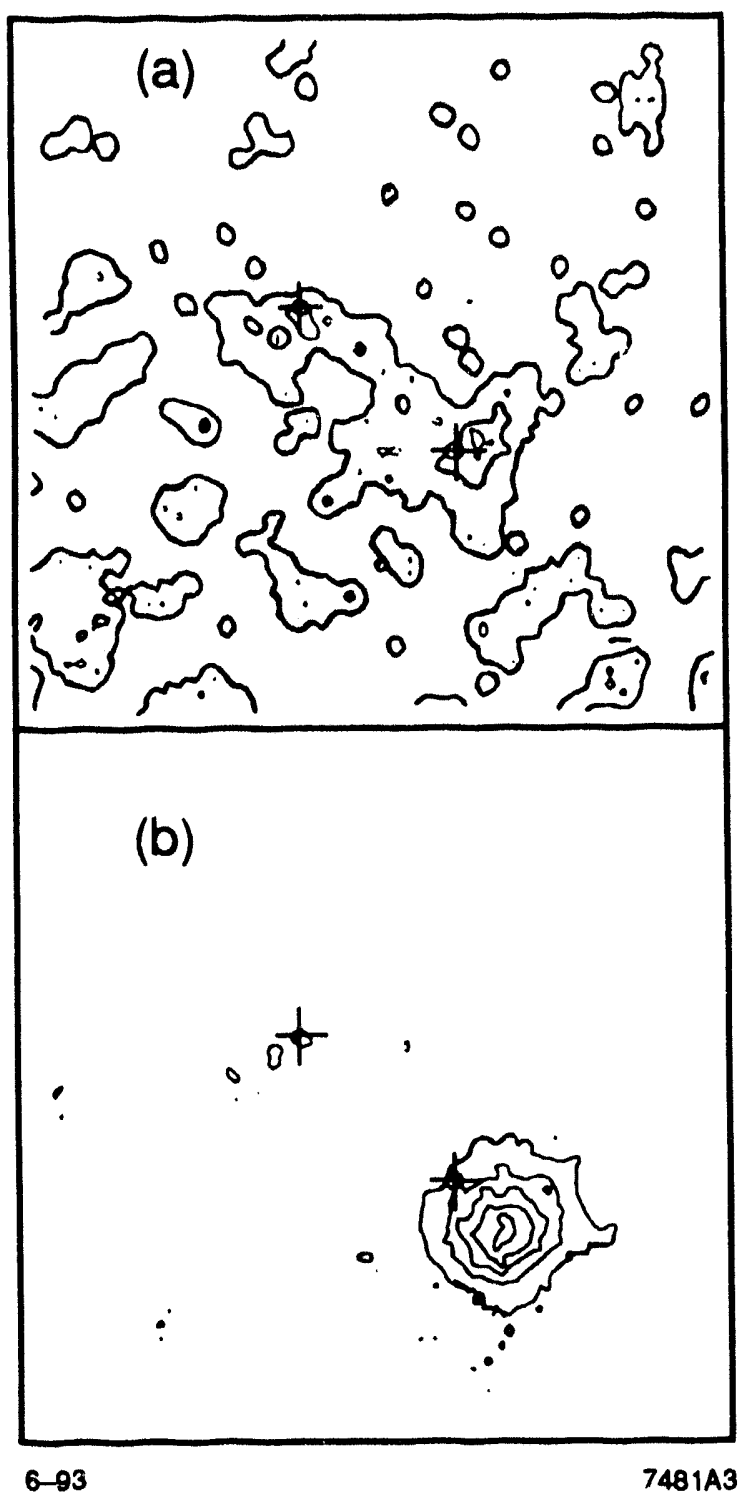

Fig. 3 


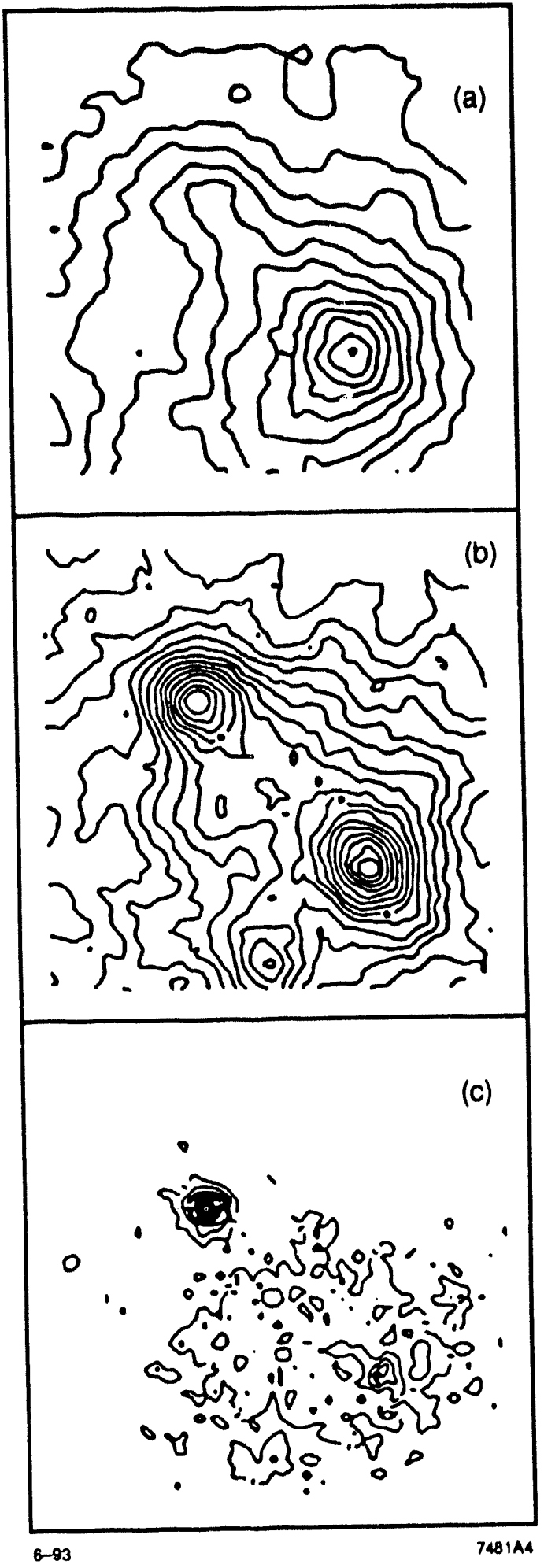

Fig. 4 


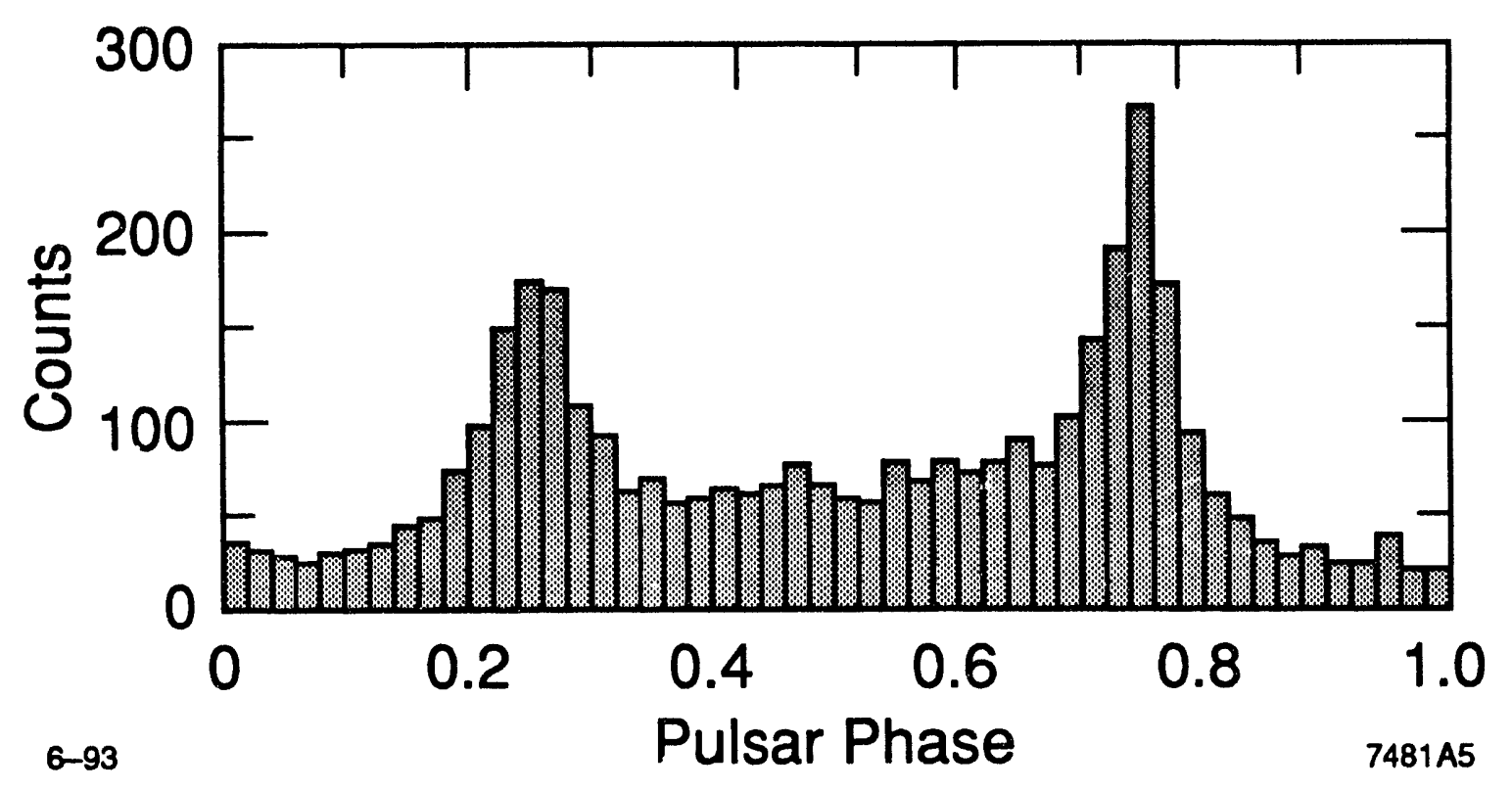

Fig. 5 


\section{Gamma Large Area Si Telesiope}

(GLAST)

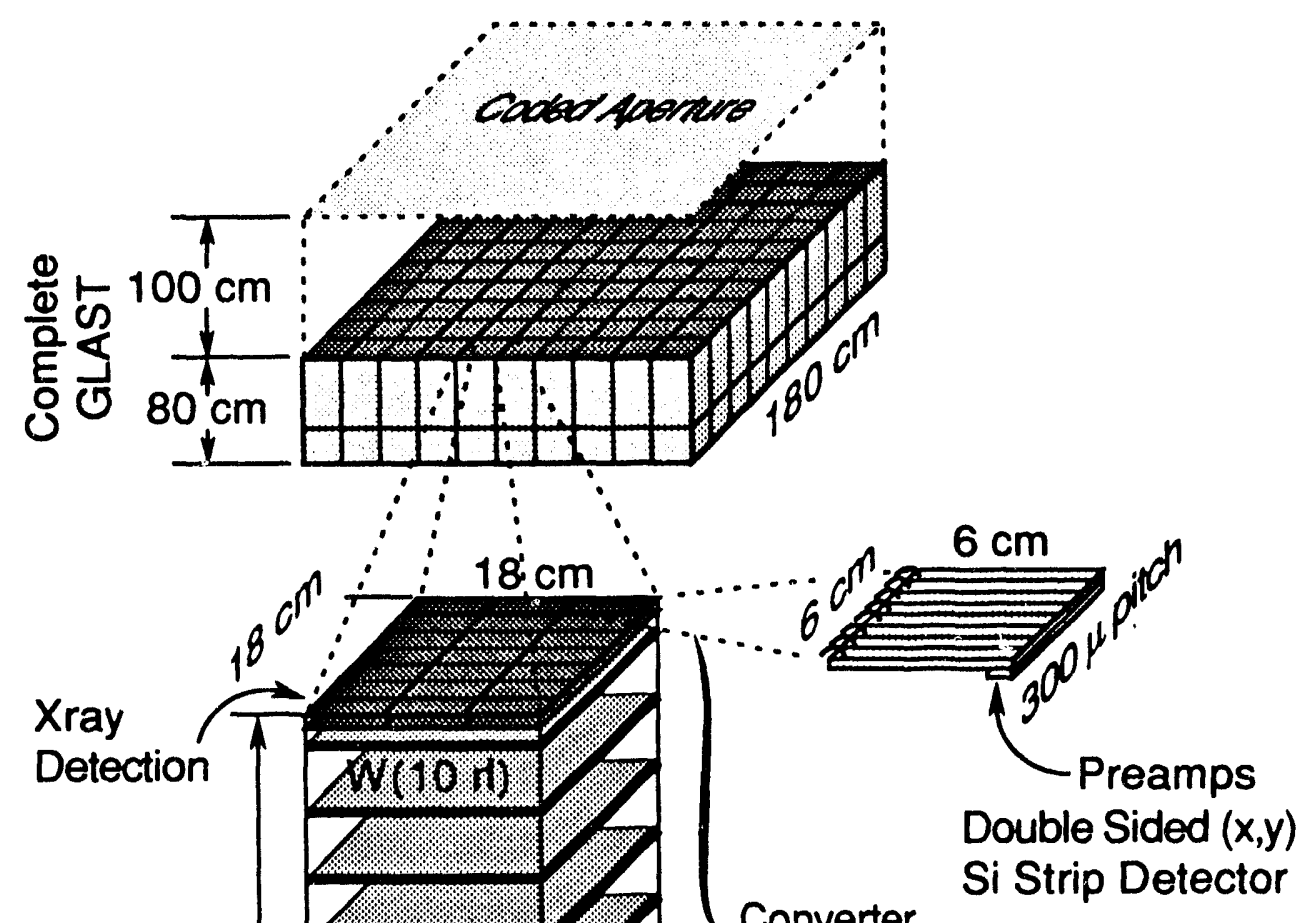

Converter

$(1.0 \mathrm{rl})$

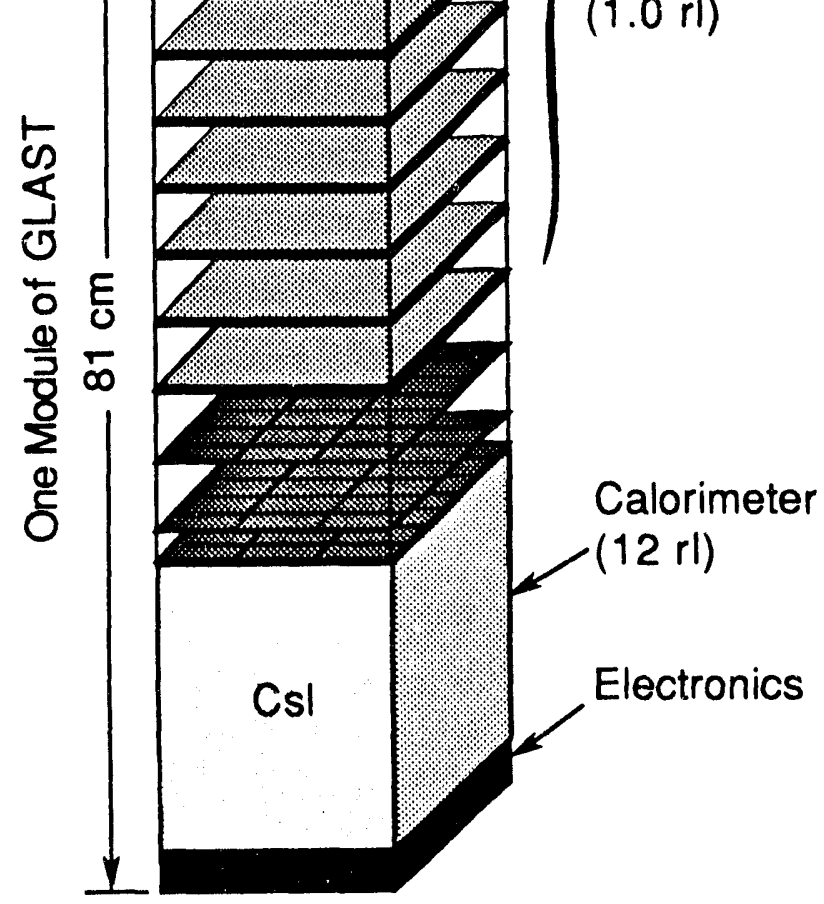

Fig. 6 


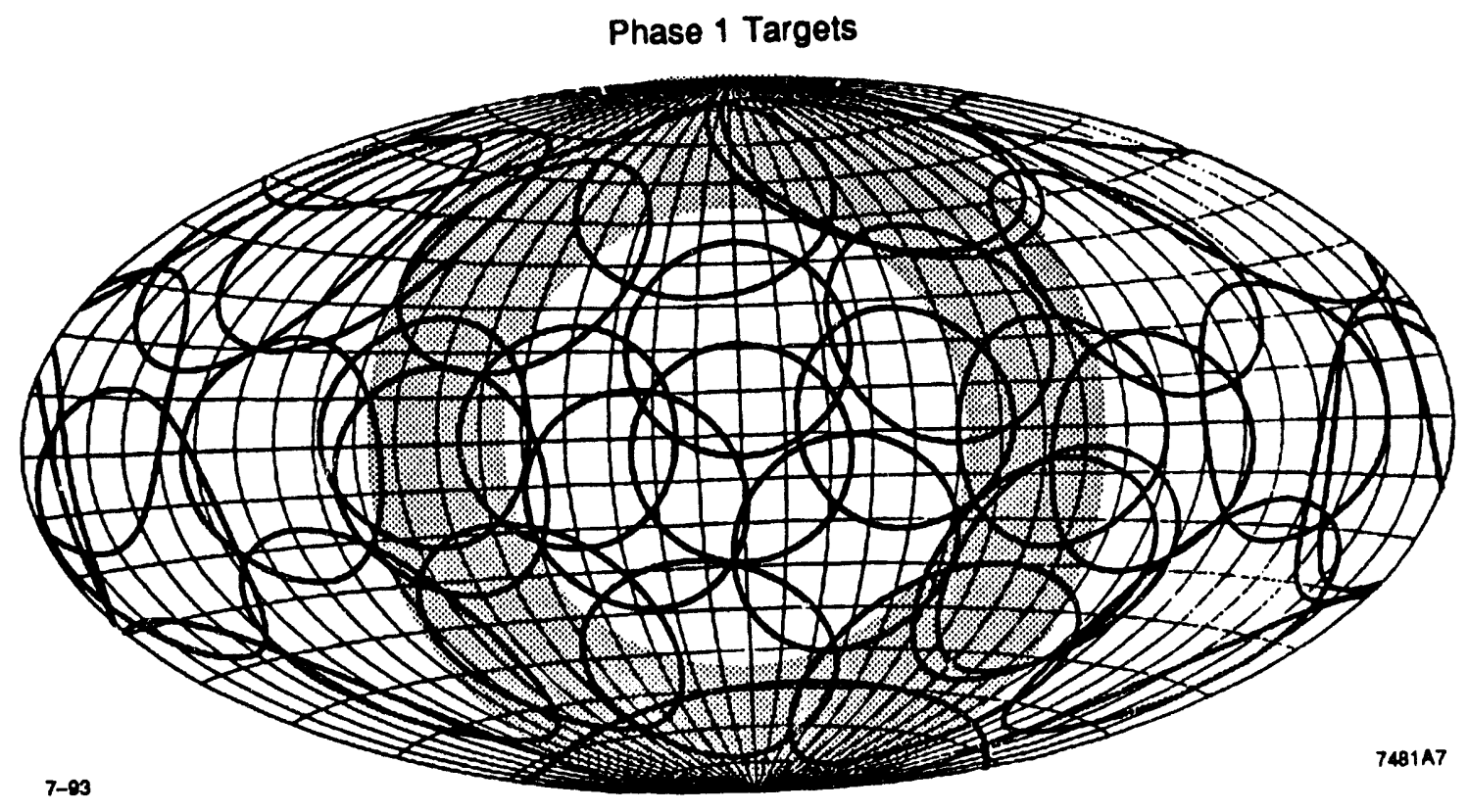

Fig. 7 

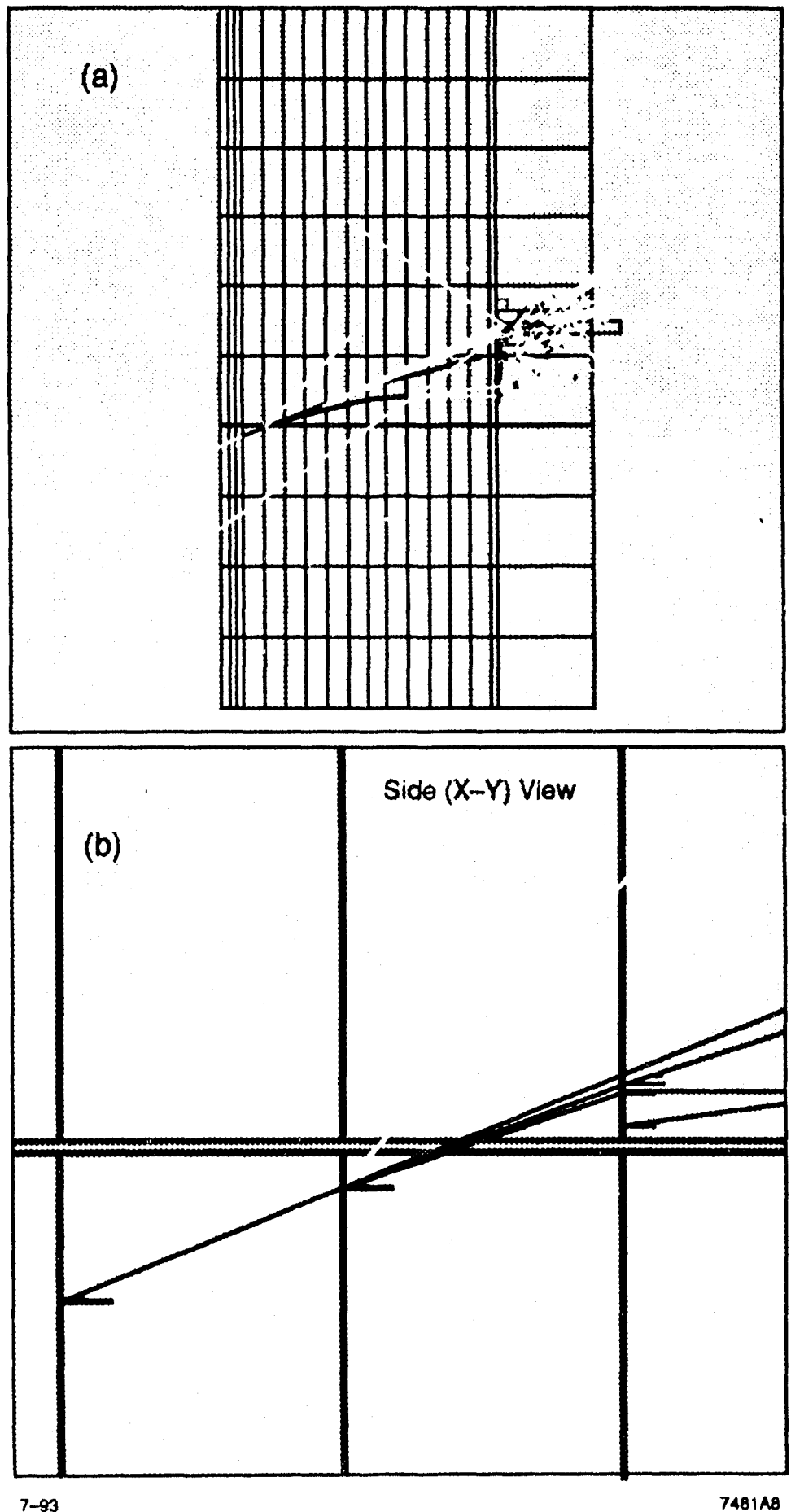

Fig. 8 

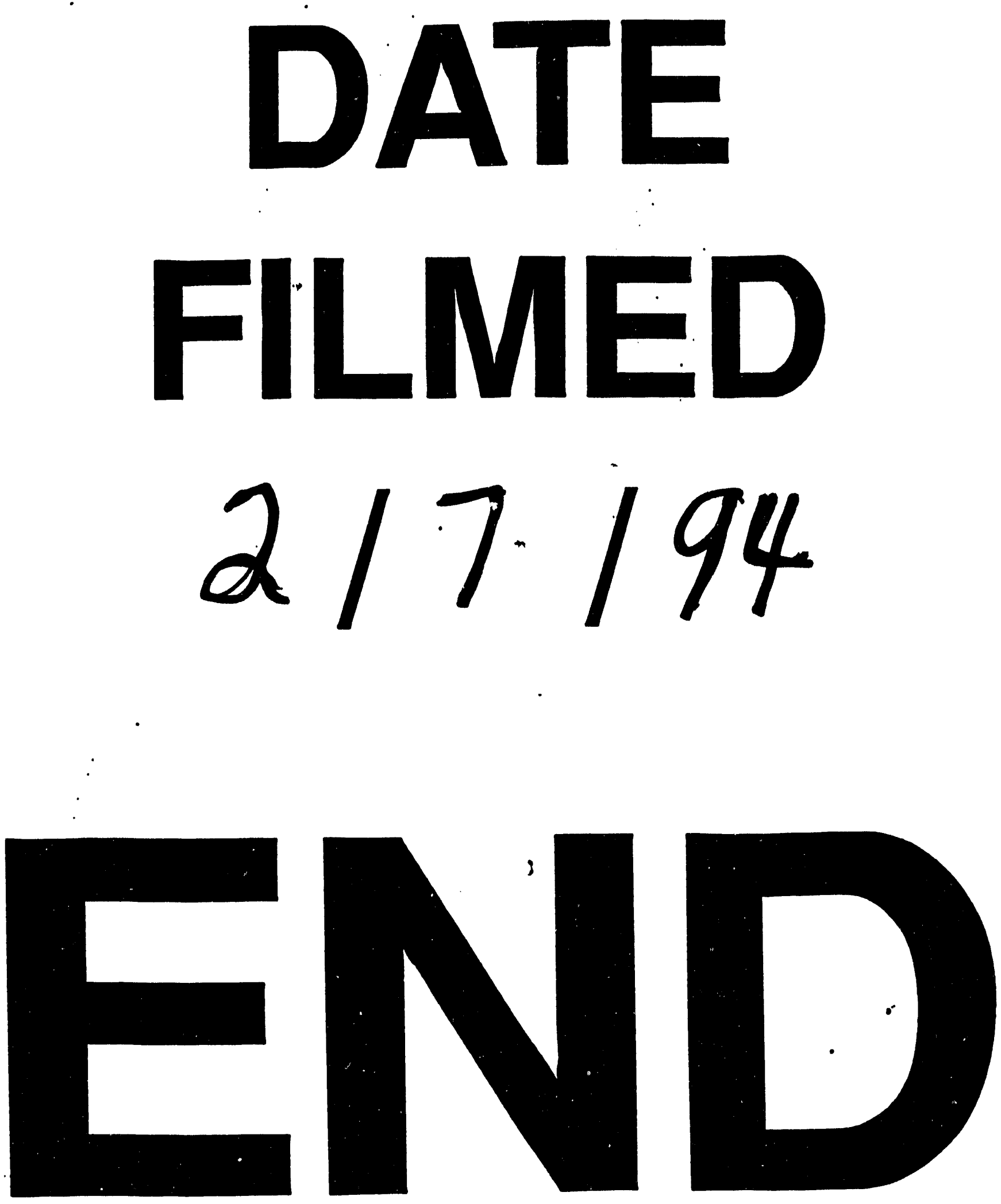


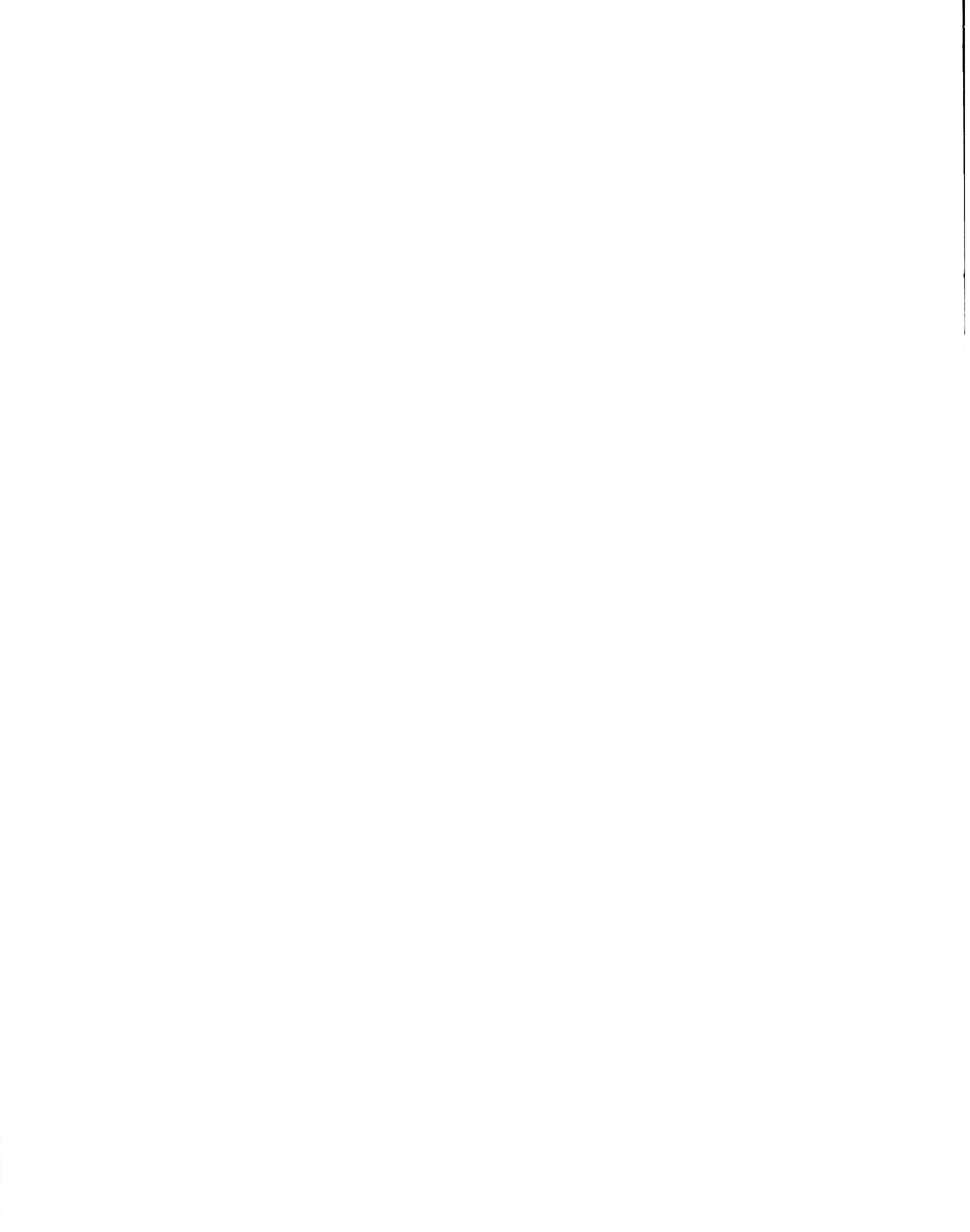

